\title{
Escherichia albertii
}

National Cancer Institute

\section{Source}

National Cancer Institute. Escherichia albertii. NCI Thesaurus. Code C86378.

A species of facultatively anaerobic, Gram-negative rod shaped bacteria assigned to the phylum Proteobacteria. This species is nonmotile, non-spore forming, catalase, lysine and ornithine decarboxylase positive, oxidase, urease, lipase, protease, and indole negative, reduces nitrate, hydrolyzes esculin, and ferments mannitol and xylose. E. albertii, originally known as Halfnia alvei, causes diarrhea. 